\section{Sarkoidose: familiäre Häufung und Erblichkeit}

Rossides $\mathrm{M}$ et al. Familial aggregation and heritability of sarcoidosis: a Swedish nested casecontrol study. Eur Respir] 2018; 52: 1800385

Die Sarkoidose wird auf eine familiäre Belastung und Umwelteinflüsse zurückgeführt. Dabei war bislang unklar, in welchem Ausmaß genetische Faktoren bedeutsam sind. Die schwedische Fall-Kontroll-Studie ermittelte die Relevanz der Faktoren mit 23880 Erkrankten und 171891 passenden Kontrollen.

Bei der systemischen Granulomatose induzieren exogene Trigger bei genetisch prädisponierten Personen eine immunologische Antwort. Verschiedene Genloci sind identifiziert, die pathophysiologisch relevant sein sollen. Frühere Studien arbeiteten mit Fragebögen an die Patienten. Die Arbeitsgruppe um Rossides fand die Erkrankten in verschiedenen schwedischen Gesundheitsregistern, die auch Informationen zum Phänotyp (Löfgren, Nicht-Löfgren) enthielten. Die Datenextraktion umfasste die Jahre 1964-2013. Die Autoren ermittelten die familiäre Häufung stratifiziert nach Geschlecht, Alter, Phänotyp, Verwandtschaftsgrad und Anzahl der betroffenen Familienmitglieder. Die Datenbanken waren Grundlage für die Ermittlung der Erblichkeit (biometrische Varianzkomponentenanalyse). Patienten < 18 Jahre und mit hämatologischen sowie Bronchialneoplasien nahmen nicht teil. Die Bedeutung des Phänotyps bestimmten die Wissenschaftler für eine Subgruppe mit $983 \mathrm{~Pa}$ tienten.

Das Durchschnittsalter der Patienten betrug 50 Jahre und $47 \%$ waren Frauen. Etwa 4\% der Betroffenen und 1\% der Kontrollen hatten 1 Verwandten 1.Grades mit Sarkoidose. Das familiäre Risiko (RR) lag bei 3,73 (95\%-Konfidenzintervall KI 3,43-4,06). Wenn 2 Verwandte 1. Grades ebenfalls erkrankt waren, stieg das relative Risiko an auf 4,69 (95\%-KI 2,93-7,51). Bei einer Sarkoidose eines Anverwandten 2. Grades war die Risikosteigerung abgeschwächt (RR 1,50; $95 \%-K I$ 0,98-2,30). Die Stratifizierung ergab Faktoren, die mit einer gesteigerten Wahrscheinlichkeit assoziiert waren:

- Alter<50 Jahre RR 3,99 vs.

RR 3,48 $\geq 50$ Jahre,

- männlicher Patient, erkrankter weiblicher Verwandter RR 4,10 vs. andere Konstellationen,

- Löfgren-Erkrankung RR 4,14 vs. 3,32 Nicht-Löfgren-Erkrankung.

Die statistische Analyse (additive genetic and nonshared environmental variance model) ergab eine Erblichkeit von 39\%, wobei das Geschlecht keinen Einfluss hatte. Eine Varianz durch geteilte Umwelteinflüsse von Geschwistern bestand nicht.

FAZIT

Die 3 Generationen umspannende Analyse belegte den Einfluss der familiären Häufung und ein bis zu 4-fach erhöhtes Risiko bei ebenfalls betroffenen Verwandten. Die Erblichkeit von $39 \%$ spricht für eine stärkere Relevanz der Umweltfaktoren als bislang angenommen. Diese gelte es zu identifizieren und therapeutisch zu nutzen, so die Autoren. Sie räumen u. a. ein, dass bei der Erblichkeitsberechnung eine Normalverteilung vorausgesetzt und eine Dominanz unter den Genloci sowie Geninteraktionen nicht angenommen wurden.

Dr. med. Susanne Krome, Melle 